\title{
Adherencia al uso de audífonos en adultos mayores del Servicio de Salud Aconcagua
}

\author{
Adherence to the use of hearing aids in elderly of the Servicio de Salud Aconcagua
}

Alex León I', Rodrigo Ediap R², Rina Carvallo T².

\section{RESUMEN}

Introducción: La prevalencia de hipoacusia en adultos mayores chilenos es de $48,5 \%$, de los cuales sólo $53 \%$ utiliza audífonos.

objetivos: Medir la adherencia al uso de audífonos y caracterizar a estos usuarios del Servicio de Salud Aconcagua.

Material y método: Se realizó un estudio descriptivo en 42 adultos mayores que recibieron audífonos durante los años 2005 a 2007, a través de revisión de fichas clínicas, observación directa y entrevista.

Resultados: Se destaca que $90 \%$ de los beneficiarios refiere utilizar el audífono y sólo $40 \%$ lo usa en el momento de la visita domiciliaria, siendo sólo $21 \%$ los que 10 hacían correctamente. Los beneficiarios adherentes residen mayormente en zonas urbanas, presentan escolaridad y nivel socioeconómico más alto que los no adherentes. Destaca que los pacientes no adherentes presentan un mayor número de comorbilidades y una menor autovalencia.

Discusión: Factores que favorecerían el uso de audífonos son: escolaridad alta, residencia urbana, experiencia previa con audífonos y percepción de gravedad de la enfermedad.

Conclusiones: El objetivo del programa de entrega de audífonos, en esta zona, sólo se cumple en $21 \%$ de los beneficiarios. La educación sobre el uso del audífono y el seguimiento mejorarían la adherencia y calidad de vida del paciente.

Palabras clave: Audífonos, adultos mayores.

\section{ABSTRACT}

Introduction: The frequency of hypoacusis in elderly is $48,5 \%$, of which only $53 \%$ uses hearing aids.

1 Médico del Servicio de Otorrinolaringología del Hospital San Camilo, tesista de Magíster en Ciencias Medicas, Mención Neurociencias de la Universidad de Chile.

2 Estudiantes de Medicina, Facultad de Medicina, Universidad de Valparaíso. SOCEMUV. 
Aim: To measure the adherence to the use of hearing aids and to characterize these users of the Servicio de Salud Aconcagua.

Material and method: A descriptive study was realized in 42 elderly who received hearing aids during the year 2005 to 2007, of review of clinical cards, direct observation and interviewed.

Results: To emphasizes that $90 \%$ of the beneficiaries mentions to use the hearing aids and only $40 \%$ to use it in the moment of the domiciliary visit, though only $21 \%$ those who were doing it correctly. The beneficiaries adherents domiciled majority in urban zones, present education and socioeconomic level higher than not adherents. It emphasizes that the patients not adherents present a major number of comorbidities and a lower autovalency.

Discussion: Factors that would favor the use of hearing aids are: high education, urban domiciled, previous experience with hearing aids and seriously perception of the disease.

Conclusions: The objective of the program of delivery of hearing aids, in this zone, only is fulfilled in $21 \%$ of the beneficiaries. The education on the use of the hearing aids and the follow-up they would improve the adherence and quality of life of the patient.

Key words: Hearing aids, elderly.

\section{INTRODUCCIÓN}

En Chile la hipoacusia afecta al $48,5 \%{ }^{1}$ de la población, siendo los adultos mayores el principal grupo afectado. La causa más frecuente es la presbiacusia ${ }^{2}$, estimándose en mayores de 75 años $40 \%$ a $50 \%{ }^{3}$, ésta es un deterioro gradual de la audición producto del envejecimiento, generalmente bilateral y simétrico.

El Fondo Nacional de Salud (FONASA) ha buscado mejorar las funcionalidades del adulto mayor ${ }^{4}$, entregando a los mayores de 65 años audífonos según la prescripción del médico otorrinolaringólogo. Desde el 1 de julio de $2007^{5}$, este beneficio se incorporó a las Garantías Explícitas en Salud, derecho que otorga la ley a todos los afiliados a FONASA e ISAPRES, con el fin de mejorar la calidad de vida y disminuir la morbimortalidad de estos pacientes ${ }^{2}$, dado que el uso de audífonos mejora su integración social, autonomía y disminuye el impacto de la hipoacusia en su salud mental, rescatando los restos auditivos y haciendo perceptibles frecuencias que sin él no son audibles.
Según la I Encuesta Nacional de Salud, la prevalencia de hipoacusia en los adultos mayores chilenos es $77,8 \%$ y la adherencia al uso de audífonos es de $53 \%{ }^{1}$. Sin embargo, este último dato fue recopilado considerando sólo lo referido por el paciente en la encuesta ${ }^{1}$. En el Servicio de Salud Aconcagua no existen datos de prevalencia de esta patología. La adherencia al uso de audífonos análogos en esta zona es de $9 \%{ }^{6}$. Debido a que actualmente se entregan audífonos digitales es indispensable renovar estos datos.

\section{OBJETIVO}

Los objetivos de este estudio descriptivo aleatorizado son medir la adherencia a esta terapia, considerando no sólo lo referido por los beneficiarios, sino también lo observado en la visita domiciliaria y caracterizarlos según su adherencia, identificando algunos factores que podrían estar influyendo en ella. 


\section{MATERIAL Y MÉTODO}

Este estudio descriptivo se llevó a cabo en el Servicio de Salud Aconcagua el año 2007 durante los meses de abril a diciembre. Los datos se recolectaron mediante una visita domiciliaria, donde se utilizó la observación del uso del audífono, sus condiciones y una encuesta confidencial, aprobada por un consentimiento informado, que indicaba el uso que se le daría a la información. Además se realizó una revisión de las fichas clínicas para conocer el grado de disminución de agudeza auditiva según la audiometría.

La muestra se obtuvo del registro de adultos mayores que recibieron audífonos entre los años 2005 y 2007, en total 250 personas, de las cuales 7 no contaban con registro domiciliario y 3 habían fallecido (información entregada por la encargada del programa). A este universo se le aplicó un muestreo aleatorio simple. El tamaño de la muestra se determinó mediante el software EPI INFO 6. Considerando las pérdidas (12), la muestra fue de 42 personas (con más del $95 \%$ de confiabilidad).

En la encuesta se utilizaron algunas preguntas de la Encuesta Nacional de Salud de 2003 y del Cuestionario Abreviado para la Evaluación de Discapacidades de FONADIS.

Los pacientes se categorizaron en:

- Adherentes: Uso diario del audífono y su uso durante la visita domiciliaria.

- No adherentes: Si no utilizaban el audífono al momento de la visita domiciliaria.

- Mal uso: Si utilizaban en la visita un audífono de otra persona, en el oído incorrecto, sin pila cargada 0 mal puesto en el conducto auditivo externo.

Se consideró como variables en estudio las siguientes: demográficas, sociales, de salud, de asistencia sanitaria y conocimiento del paciente sobre su enfermedad.

Se realizó un análisis estadístico descriptivo de los datos mediante el software EPI INFO 6.

\section{RESULTADOS}

Con respecto a la adherencia al uso de audífonos se encontró lo siguiente:

El $90 \%$ de la muestra (38 personas) refiere utilizar el audífono. El $43 \%$ de éstos refiere utilizarlo todos los días y el $48 \%$ varias veces a la semana o en ocasiones especiales; de estos últimos el $80 \%$ sólo ha gastado $\leq 3$ pilas.

El $40 \%$ (17) del total utiliza el audífono en la visita domiciliaria. Sin embargo, $47 \%$ (8) de éstos, lo hacen de manera errónea. Del $60 \%$ que no utiliza el audífono en la visita, $72 \%$ ha gastado $\leq 3$ pilas.

El número de pilas utilizado no presenta relación con la edad de los pacientes, pero sí con el grado de hipoacusia. Cuando la hipoacusia es leve el promedio de pilas es de 0,8; si es moderada, el promedio es de 2 y si es severa, el promedio de pilas asciende a 3,4 .

La Tabla 1 muestra que en las variables socioeconómicas hay importantes diferencias entre quienes usan el audífono y quienes no lo usan. Por ejemplo, la residencia urbana es mayor en los beneficiarios adherentes, lo mismo ocurre con la escolaridad y el nivel socioeconómico medio-alto.

Con respecto a la variable salud, destaca que los pacientes no adherentes presentan un mayor número de comorbilidades y una menor autovalencia.

En cuanto a asistencia sanitaria, resalta la baja accesibilidad al centro de referencia para la entrega de la prótesis presentada por los beneficiarios que no utilizan el audífono o lo hacen erróneamente.

Finalmente, el conocimiento respecto de la hipoacusia y del manejo del audífono es menor en los pacientes no adherentes y en quienes efectúan un mal uso del audífono.

\section{DISCUSIÓN}

Considerando que la adherencia al tratamiento es un proceso multifactorial, parece ser que algunos factores que favorecerían el uso de audífonos son: 
Tabla 1. Principales características de los beneficiarios de audífonos. Valle de Aconcagua, 2007

\begin{tabular}{|c|c|c|c|c|c|}
\hline \multirow{2}{*}{$\begin{array}{l}\text { Variables en estudio } \\
\text { Socio-demográficas }\end{array}$} & \multicolumn{2}{|c|}{ Características } & \multirow{2}{*}{$\begin{array}{c}\text { Adherentes } \\
56\end{array}$} & \multirow{2}{*}{$\begin{array}{c}\text { No adherentes } \\
48\end{array}$} & \multirow{2}{*}{$\frac{\text { Mal uso }}{40}$} \\
\hline & $\operatorname{Sexo}(\%)$ & Masculino & & & \\
\hline & & Femenino & 44 & 52 & 60 \\
\hline & \multicolumn{2}{|c|}{ Residencia urbana (\%) } & 89 & 52 & 50 \\
\hline & \multicolumn{2}{|c|}{ Escolaridad (años) } & 13 & 4 & 4 \\
\hline & \multicolumn{2}{|c|}{ Analfabetismo (\%) } & 0 & 25 & 33 \\
\hline & \multirow[t]{4}{*}{$\operatorname{NSE}^{*}(\%)$} & Bajo & 11 & 80 & 100 \\
\hline & & Medio-bajo & 11 & 12 & 0 \\
\hline & & Medio & 33 & 4 & 0 \\
\hline & & Medio-alto & 44 & 4 & 0 \\
\hline & \multicolumn{2}{|c|}{ Ayuda familiar en manejo audífono (\%) } & 11 & 56 & 50 \\
\hline \multirow[t]{5}{*}{ Salud } & \multicolumn{2}{|c|}{ № enfermedades crónicas } & $\leq 2$ & $\geq 3$ & $\leq 3$ \\
\hline & \multicolumn{2}{|c|}{ Autovalencia $^{\star *}(\%)$} & 89 & 60 & 63 \\
\hline & \multicolumn{2}{|c|}{ Percepción hipoacusia sin audífono } & Severa & Leve-moderada & Severa \\
\hline & \multicolumn{2}{|c|}{ Percepción hipoacusia con audífono } & Leve-moderada & Severa & Severa \\
\hline & \multicolumn{2}{|c|}{ Cambio positivo en su vida (\%) } & 78 & 36 & 63 \\
\hline \multirow[t]{2}{*}{ Asistencia sanitaria } & \multicolumn{2}{|c|}{ Uso previo permanente ${ }^{* * *}(\%)$} & 80 & 17 & 80 \\
\hline & \multicolumn{2}{|c|}{$\begin{array}{l}\text { Accesibilidad baja al centro de } \\
\text { referencia }^{* \star *}(\%)\end{array}$} & 33 & 52 & 50 \\
\hline Conocimiento & \multirow{2}{*}{\multicolumn{2}{|c|}{$\begin{array}{l}\text { Inadecuado sobre hipoacusia (\%) } \\
\text { Nulo sobre el manejo audífono (\%) }\end{array}$}} & 44 & 80 & 75 \\
\hline del paciente ${ }^{\star * * * *}$ & & & 11 & 92 & 75 \\
\hline Variables en estudio & \multicolumn{2}{|c|}{ Características } & Adherentes & No adherentes & Mal uso \\
\hline \multirow[t]{10}{*}{ Socio-demográficas } & \multirow[t]{2}{*}{$\operatorname{Sexo}(\%)$} & Masculino & 56 & 48 & 40 \\
\hline & & Femenino & 44 & 52 & 60 \\
\hline & \multicolumn{2}{|c|}{ Residencia urbana (\%) } & 89 & 52 & 50 \\
\hline & \multicolumn{2}{|c|}{ Escolaridad (años) } & 13 & 4 & 4 \\
\hline & \multicolumn{2}{|c|}{ Analfabetismo (\%) } & 0 & 25 & 33 \\
\hline & $\operatorname{NSE}^{*}(\%)$ & Bajo & 11 & 80 & 100 \\
\hline & & Medio-bajo & 11 & 12 & 0 \\
\hline & & Medio & 33 & 4 & 0 \\
\hline & & Medio-alto & 44 & 4 & 0 \\
\hline & Ayuda fam & en manejo audífono (\%) & 11 & 56 & 50 \\
\hline Salud & $N^{\circ}$ Enferm & des crónicas & $\leq 2$ & $\geq 3$ & $\leq 3$ \\
\hline & Autovaleno & * $(\%)$ & 89 & 60 & 63 \\
\hline & Percepción & poacusia sin audífono & Severa & Leve-moderada & Severa \\
\hline & Percepción & poacusia con audífono & Leve-moderada & Severa & Severa \\
\hline & Cambio po & vo en su vida (\%) & 78 & 36 & 63 \\
\hline Asistencia sanitaria & Uso previo & rmanente $^{\star \star \star}(\%)$ & 80 & 17 & 80 \\
\hline & $\begin{array}{l}\text { Accesibilid } \\
\text { referencia* }\end{array}$ & $\begin{array}{l}\text { baja al centro de } \\
\text { * }(\%)\end{array}$ & 33 & 52 & 50 \\
\hline Conocimiento & Inadecuad & bre hipoacusia (\%) & 44 & 80 & 75 \\
\hline del paciente $e^{\star \star \star \star \star}$ & Nulo sobre & manejo audífono (\%) & 11 & 92 & 75 \\
\hline
\end{tabular}

* Nivel socioeconómico (NSE) se basó "Metodología para agrupar establecimientos según nivel socioeconómico".

* * Se extrajeron preguntas del "Cuestionario Abreviado para la Evaluación de Discapacidades de FONADIS" para evaluarla.

*** Presentar una prescripción previa de audífono y su uso diario por más de 1 mes.

**** Se evaluó de acuerdo al tiempo y dinero utilizado por el beneficiario en el traslado desde su domicilio al establecimiento donde se desarrolla el programa.

***** Se realizó varias preguntas sobre ambos aspectos y cada una de ellas se le dio un puntaje, de acuerdo a la sumatoria de éstas se categorizó el conocimiento. 
escolaridad alta, residencia urbana y nivel socioeconómico (NSE) familiar medio-alto, además de la experiencia de uso de audífonos previa y la percepción del paciente de la gravedad de su enfermedad.

Las molestias con el uso de audífonos son comunes entre los usuarios, siendo la más frecuente la amplificación molesta, lo que, en general, correspondería a la gran cantidad de ruidos que posee el ambiente. El $26 \%$ de los beneficiarios presenta este problema y no usan el audífono. Resultados similares a lo estimado por el MINSAL, quien refiere que el $25 \%{ }^{1}$ no lo utiliza debido a esta causa, sin embargo, en este caso, no se puede establecer causalidad.

La existencia de roles diferenciados de género adoptados en el cuidado de la salud de la familia 0 en la pareja ${ }^{7}$, podría explicar la menor adherencia presentada por el sexo femenino, quienes se ven muy poco apoyadas en el cuidado de su salud.

Dada las características de este estudio, la descripción realizada sólo pretende entregar antecedentes que permitan identificar aquellos factores modificables con el objeto de generar estrategias más efectivas para mejorar la adherencia al uso de audífonos.

\section{CONCLUSIONES}

En el Servicio de Salud Aconcagua, utilizando Ios mismos parámetros de la Encuesta Nacional de Salud, se obtiene $90 \%$ de adherencia, mayor al $53 \%$ encontrado a nivel nacional y al $79,2 \%$ encontrado en Santiago por Tamblay y cols ${ }^{8}$.

Al aplicar la observación este valor disminuye a $40 \%$, existiendo entonces $60 \%$ de pacientes no adherentes a la prótesis, cifra que se asemeja a lo encontrado en el estudio "Adherencia al tratamiento" $(59 \%)^{9}$, realizado en Colombia.

Al verificar cómo era utilizado el audífono, el $19 \%$ lo hacía incorrectamente, entonces el objetivo del programa de entrega de audífonos, en esta zona, sólo se cumple en el $21 \%$ de los beneficiarios.

Los pacientes que usan el audífono diariamente refieren presentar un cambio positivo en su vida, lo que coincide con lo concluido en el estudio de Tamblay antes mencionado ${ }^{8}$.

Al excluir de los adherentes aquellos casos en que esta terapia no presenta mejoría de la calidad de vida, se obtiene que el uso beneficioso de audífonos corresponde a sólo $17 \%$ menor al $66 \%$ esperado en la Guía Clínica del Ministerio de Salud (MINSAL) ${ }^{1}$

Considerando los resultados obtenidos, la evidencia internacional y nacional y las recomendaciones de la guía clínica antes mencionada, la educación sobre el uso del audífono y el seguimiento mejorarían la adherencia y calidad de vida del paciente.

Se debe enfatizar que los beneficiarios que utilizan erróneamente el audífono presentan interés por el uso de su prótesis, por lo que con una buena educación presentarían una mejor adherencia.

\section{BIBLIOGRAFÍA}

1. Departamento de Salud Pública PUC. Resultados I Encuesta de Salud, Chile 2003 [Internet]. Santiago: Minsal; 2004 [acceso 30 de abril de 2007].

2. Ministerio de Salud. Guía Clínica Hipoacusia Bilateral en personas de 65 años y más que requieren uso de audífono [Internet]. Santiago: Minsal; 2007 [acceso 26 de diciembre de 2007].

3. Centro ORL Avanzada, Presbiacusia [internet]. Madrid: Coagavilan.es [acceso 1 de marzo de 2010].

4. Fonasa.cl, Programa Adulto Mayor [internet]. Santiago: Fonosa.cl [acceso 20 de diciembre de 2007].

5. Ministerio de Salud y Ministerio de Hacienda. Decreto $N^{0} 44$, Tercer Régimen de Garantías Explícitas en Salud [Internet]. Santiago: Minsal; 2007.

6. Herrera I, Rivera Y. Informe Final, Evaluación Sistema de entrega de audífonos a beneficiarios 1995-2001, Programa Adulto Mayor [tesis]. Valparaíso: Universidad de Valparaíso; 2002.

7. Mendoza S, Muñoz M, Merino J, Barriga 0. Factores determinantes de cumplimiento tera- 
péutico en adultos mayores hipertensos. Rev Méd Chile 2006; 134(1): 65-71.

8. Tamblay N, Villalobos I, Pastene A, Rahal M. Impacto social del uso de audífonos en adultos mayores. Rev Otorrinolaringol Cir
Cabeza Cuello 2008; 68: 21-6.

9. Silva Ge, Galeano E, Correa J0. Adherencia al tratamiento: Implicaciones de la no-adherencia. Acta Médica Colombiana 2005; 30 (4): 268-73. 